\title{
Contractible Lie Groups over Local Fields
}

\author{
Helge Glöckner*
}

\begin{abstract}
Let $G$ be a Lie group over a local field of characteristic $p>0$ which admits a contractive automorphism $\alpha: G \rightarrow G$ (i.e., $\alpha^{n}(x) \rightarrow 1$ as $n \rightarrow \infty$, for each $x \in G$ ). We show that $G$ is a torsion group of finite exponent and nilpotent. We also obtain results concerning the interplay between contractive automorphisms of Lie groups over local fields, contractive automorphisms of their Lie algebras, and positive gradations thereon. Some of the results even extend to Lie groups over arbitrary complete ultrametric fields.
\end{abstract}

Classification: 22E20 (primary), 20E15, 20E36, 26E30, 37D10

Key words: Lie group, local field, ultrametric field, positive characteristic, contraction group, contractive automorphism, contractible group, torsion group, positive gradation, nilpotent group, stable manifold, Lie subgroup, composition series

\section{Introduction}

A contraction group is a pair $(G, \alpha)$ of a topological group $G$ and a (bicontinuous) automorphism $\alpha: G \rightarrow G$ which is contractive in the sense that $\alpha^{n}(x) \rightarrow 1$ as $n \rightarrow \infty$, for each $x \in G$. It is known from the work of Siebert 28] that each locally compact contraction group is a direct product $G=G_{0} \times H$ of its identity component $G_{0}$ and an $\alpha$-stable totally disconnected group $H$. Siebert also showed that $G_{0}$ is a simply connected, nilpotent real Lie group. Results concerning the totally disconnected part $H$ were obtained in [18]. It is a direct product

$$
H=H_{p_{1}} \times \cdots \times H_{p_{n}} \times \operatorname{tor}(H)
$$

of its subgroup tor $(H)$ of torsion elements and certain $\alpha$-stable $p$-adic Lie groups $H_{p}$. Thus $p$-adic contraction groups are among the basic building blocks of general contraction groups, and it is therefore well motivated

* Research supported by the German Research Foundation (DFG), grant GL 357/6-1 
to study these, and more generally contraction groups which are (finitedimensional) Lie groups over local fields. Essential structural information concerning $p$-adic contraction groups was obtained by Wang [30]: He showed that any such is a unipotent algebraic group defined over $\mathbb{Q}_{p}$ (and hence nilpotent). The main goal of the current article is to shed light on contraction groups which are Lie groups over local fields of positive characteristic.

Theorem A. Let $G$ be a $C^{1}$-Lie group over a local field $\mathbb{K}$ of characteristic $p>0$ which admits a contractive $C^{1}$-automorphism $\alpha: G \rightarrow G$. Then $G$ is a torsion group of finite exponent and solvable. Furthermore, there exists a series

$$
\mathbf{1}=G_{0} \triangleleft G_{1} \triangleleft \cdots \triangleleft G_{n}=G
$$

of $\alpha$-stable, closed subgroups $G_{j}$ such that the contraction group $G_{j} / G_{j-1}$ is isomorphic to $C_{p}^{(-\mathbb{N})} \times C_{p}^{\mathbb{N}_{0}}$ with the right shift, for each $j \in\{1, \ldots, n\}$.

Here $C_{p}$ is the cyclic group of order $p$ and $C_{p}^{(-\mathbb{N})} \times C_{p}^{\mathbb{N}_{0}}$ the restricted product of all functions $f: \mathbb{Z} \rightarrow C_{p}$ such that $f(n)=1$ for $n$ below some $n_{0}$, with the infinite power $C_{p}^{\mathbb{N}_{0}}$ as a compact open subgroup. The right shift $\sigma$ is defined via $\sigma(f)(n):=f(n-1)$, and a morphism between contraction groups $\left(G_{1}, \alpha_{1}\right)$ and $\left(G_{2}, \alpha_{2}\right)$ is a continuous homomorphism $\phi: G_{1} \rightarrow G_{2}$ such that $\alpha_{2} \circ \phi=\phi \circ \alpha_{1}$. The series (1) is a composition series of topological $\langle\alpha\rangle$-groups (in the sense of [18]).

We are mainly interested in $\mathbb{K}$-analytic $\left(C^{\omega}\right.$-) Lie groups and $\mathbb{K}$-analytic automorphisms, but the preceding result holds just as well for $C^{1}$-Lie groups and their automorphisms, and has been formulated accordingly. Recall that, while $C^{k}$-Lie groups and analytic Lie groups coincide in the $p$-adic case [10], for each local field of positive characteristic there exist non-analytic smooth Lie groups and $C^{k}$-Lie groups which are not $C^{k+1}$, for each $k \in \mathbb{N}$ (see [9]).

Our second main result says that $G$ is not only solvable, but nilpotent, at least under a slightly stronger differentiability hypothesis $(k \geq 2)$. The conclusion even remains valid for Lie groups over complete ultrametric fields which are not necessarily locally compact.

Theorem B. Given $k \in \mathbb{N} \cup\{\infty, \omega\}$ with $k \geq 2$, let $G$ be a $C^{k}$-Lie group over a complete ultrametric field $(\mathbb{K},||$.$) and \alpha: G \rightarrow G$ be a contractive $C^{k}$-automorphism. Then $G$ is nilpotent. Furthermore, there exists a central 
series

$$
\mathbf{1}=G_{0} \triangleleft G_{1} \triangleleft \cdots \triangleleft G_{m}=G
$$

such that each $G_{j}$ is an $\alpha$-stable $C^{k}$-Lie subgroup of $G$.

While the proof of Theorem A (given in Section 2) is based on the structure theory of totally disconnected, locally compact contraction groups from [18], Theorem B (proved in Section 4) relies on entirely different methods: it uses the ultrametric stable manifolds constructed in [14].

To enable successful application of the methods from [14], we first take a closer look at the linearization $L(\alpha)=T_{1}(\alpha)$ of $\alpha$ around its fixed point 1 (see Section 3). For example, it is essential for us that $L(\alpha)$ is a contractive automorphism of $L(G)=T_{1}(G)$, and that each eigenvalue of $L(\alpha)$ (in an algebraic closure) has absolute value $<1$ (as shown in [14]). Further results concerning contractive Lie algebra automorphisms take Siebert's treatment of the real case as a model. He showed that each contractive automorphism of a real Lie algebra $\mathfrak{g}$ gives rise to a positive gradation on $\mathfrak{g}$, i.e., $\mathfrak{g}=\bigoplus_{r>0} \mathfrak{g}_{r}$ for vector subspaces $\mathfrak{g}_{r} \subseteq \mathfrak{g}$ indexed by positive reals such that $\mathfrak{g}_{r}=\{0\}$ for all but finitely many $r$ and $\left[\mathfrak{g}_{r}, \mathfrak{g}_{s}\right] \subseteq \mathfrak{g}_{r+s}$ for all $r, s>0$. Conversely, each positive gradation yields contractive Lie algebra automorphisms of $\mathfrak{g}$ (see [28]).

In the case of Lie algebras over local fields, the right class of positive gradations to look at are $\mathbb{N}$-gradations, i.e. positive gradations $\mathfrak{g}=\bigoplus_{r>0} \mathfrak{g}_{r}$ such that $\mathfrak{g}_{r} \neq\{0\}$ implies $r \in \mathbb{N}$, and thus $\mathfrak{g}=\bigoplus_{r \in \mathbb{N}} \mathfrak{g}_{r}$. We show that a Lie algebra $\mathfrak{g}$ over a local field admits an $\mathbb{N}$-gradation if and only if it admits a contractive Lie algebra automorphism (Proposition 3.1).

In Section 5, we discuss the interplay between contractive automorphisms of Lie groups and Lie algebras. In the real case, it is known that each Lie group $G$ admitting a contractive automorphism $\alpha$ is simply connected, and that $L(\alpha)$ is a contractive Lie algebra automorphism. Conversely, each contractive Lie algebra automorphism of a real Lie algebra integrates to a contractive automorphism of the corresponding simply connected real Lie group. It is quite interesting that, likewise, we can always pass from the Lie algebra level to the group level in the case of complete ultrametric fields of characteristic 0 :

Theorem C. Let $(\mathbb{K},||$.$) be a complete ultrametric field of characteristic 0$, $\mathfrak{g}$ be a Lie algebra over $\mathbb{K}$ and $\beta: \mathfrak{g} \rightarrow \mathfrak{g}$ be a contractive Lie algebra auto- 
morphism. Then there exists a $\mathbb{K}$-analytic Lie group $G$, unique up to isomorphism, and a uniquely determined $\mathbb{K}$-analytic contractive automorphism $\alpha$ of $G$ such that $L(\alpha)=\beta$.

In this case, the appropriate substitute for a simply connected group is constructed with the help of an HNN-extension. Related results are also obtained if $\operatorname{char}(\mathbb{K})>0$, but these are by necessity weaker. For instance, it may happen in positive characteristic that two non-isomorphic contraction groups give rise to the same Lie algebra and the same contractive Lie algebra automorphism. An example for this phenomenon (Example 6.7) and examples illustrating various other aspects of the theory are compiled in Section 6.

Let us mention in closing that results concerning contraction groups also extend our knowledge of more general automorphisms of Lie groups over local fields. In fact, let $G$ be a $C^{k}$-Lie group over a local field, where $k \in \mathbb{N} \cup\{\infty, \omega\}$, and $\alpha: G \rightarrow G$ be an automorphism of $C^{k}$-Lie groups. Let $U_{\alpha}$ be the group of all $x \in G$ such that $\alpha^{n}(x) \rightarrow 1$ as $n \rightarrow \infty$, and $M_{\alpha}$ be the group of all $x \in G$ the two-sided orbit $\alpha^{\mathbb{Z}}(x)$ of which is relatively compact in $G$. Then $\left(U_{\alpha},\left.\alpha\right|_{U_{\alpha}}\right)$ and $\left(U_{\alpha^{-1}},\left.\alpha^{-1}\right|_{U_{\alpha^{-1}}}\right)$ are contraction groups in the induced topology, but they are also contraction groups (with contractive $C^{k}$-automorphisms) when equipped with suitable immersed $C^{k}$ Lie subgroup structures (see [14]) 1] which may correspond to properly finer topologies. Strongest results are available if $U_{\alpha}$ is closed 2 Then all of $U_{\alpha}$, $U_{\alpha^{-1}}$ and $M_{\alpha}$ are $C^{k}$-Lie subgroups of $G$, their complex product $U_{\alpha} M_{\alpha} U_{\alpha^{-1}}$ is an open $\alpha$-stable identity neighbourhood in $G$, and the product map

$$
U_{\alpha} \times M_{\alpha} \times U_{\alpha^{-1}} \rightarrow U_{\alpha} M_{\alpha} U_{\alpha^{-1}}
$$

is a $C^{k}$-diffeomorphism (see [30] for the $p$-adic case, [15] for the general result). The theorems of the current article then apply to $\left(U_{\alpha},\left.\alpha\right|_{U_{\alpha}}\right)$ and $\left(U_{\alpha^{-1}},\left.\alpha^{-1}\right|_{U_{\alpha^{-1}}}\right)$. Some basic information on $M_{\alpha}$ can be drawn from [16] (cf. [17] and [24] for the $p$-adic case): $M_{\alpha}$ has small $\alpha$-stable compact open subgroups. In contrast to the case of contraction groups, $M_{\alpha}$ need not have special group-theoretic properties: Choosing $\alpha=\mathrm{id}$, we get $G=M_{\alpha}$ and conclude that $M_{\alpha}$ can be an arbitrary $C^{k}$-Lie group.

\footnotetext{
${ }^{1}$ Making them the stable manifold and unstable manifold of $\alpha$ around 1, respectively.

${ }^{2}$ This condition is automatically satisfied if $\operatorname{char}(\mathbb{K})=0$ (see [30]) or if there exists an injective, continuous homomorphism from $G$ to a general linear group [15]. Various characterizations of closedness of $U_{\alpha}$ were given in [1].
} 
Contraction groups of the form $U_{\alpha}$ arise in many contexts: In representation theory in connection with the Mautner phenomenon (see [23, Chapter II, Lemma 3.2] and (for the $p$-adic case) [30]); in probability theory on groups (see [19], [28] and (for the $p$-adic case) [6]); and in the structure theory of totally disconnected, locally compact groups developed in [33] (see [1]).

Acknowledgement. The author thanks George A. Willis for useful discussions, notably concerning the examples in Section 6 .

\section{General conventions and facts}

Complementing the definitions already given in the Introduction, we now fix additional notation and terminology.

1.1 Conventions concerning valued fields. By a local field, we mean a totally disconnected, non-discrete locally compact topological field. We fix an ultrametric absolute value $||:. \mathbb{K} \rightarrow[0, \infty[$ on $\mathbb{K}$ defining its topology [31]. A field $\mathbb{K}$, equipped with an ultrametric absolute value $|$.$| which defines a non-$ discrete topology on $\mathbb{K}$ is called an ultrametric field; it is called complete if $\mathbb{K}$ is a complete metric space with respect to the metric $(x, y) \mapsto|y-x|$. Given a complete ultrametric field $(\mathbb{K},||$.$) (e.g., a local field), we fix an algebraic$ closure $\overline{\mathbb{K}}$ of $\mathbb{K}$ and use the same symbol, |.|, for the unique extension of the given absolute value $|$.$| to an absolute value on \overline{\mathbb{K}}$ (see [26, Theorem 15.1]). If $(E,\|\|$.$) is a normed space over a valued field (\mathbb{K},||$.$) , given x \in E$ and

$r>0$ we write $B_{r}^{E}(x):=\{y \in E:\|y-x\|<r\}$ and $B_{r}:=B_{r}^{E}(0)$. Given a continuous linear map $\alpha$ between normed spaces $\left(E,\|\cdot\|_{E}\right)$ and $\left(F,\|\cdot\|_{F}\right)$, its operator norm is defined as

$$
\|\alpha\|_{\mathrm{op}}:=\min \left\{r \in \left[0, \infty\left[:(\forall x \in E)\|\alpha(x)\|_{F} \leq r\|x\|_{E}\right\}\right.\right.
$$

1.2 Differential calculus, manifolds and Lie groups. All manifolds, Lie groups and Lie algebras considered in this article are finite-dimensional. Basic references for analytic manifolds and analytic Lie groups over complete ultrametric fields are [27], also [3] and [4]. We use the symbol " $C$ " shorthand for "analytic" and agree that $n<\infty<\omega$ for all $n \in \mathbb{N}_{0}$, where $\mathbb{N}=\{1,2, \ldots\}$ and $\mathbb{N}_{0}=\mathbb{N} \cup\{0\}$. Let $E$ and $F$ be (Hausdorff) topological vector spaces over a non-discrete topological field $\mathbb{K}$ and $U \subseteq E$ be open. Then $U^{[1]}:=\{(x, y, t) \in U \times E \times \mathbb{K}: x+t y \in U\}$ is an open subset of 
$E \times E \times \mathbb{K}$. Following [2], we say that $f$ is $C^{1}$ if it is continuous and there exists a (necessarily unique) continuous map $f^{[1]}: U^{[1]} \rightarrow F$ which extends the directional difference quotient map, i.e.,

$$
f^{[1]}(x, y, t)=\frac{f(x+t y)-f(x)}{t}
$$

for all $(x, y, t) \in U^{[1]}$ such that $t \neq 0$. Then $f^{\prime}(x):=f^{[1]}(x, \bullet, 0): E \rightarrow F$ is a continuous linear map. Inductively, $f$ is called $C^{k+1}$ for $k \in \mathbb{N}$ if $f$ is $C^{1}$ and $f^{[1]}$ is $C^{k}$. As usual, $f$ is called $C^{\infty}$ or smooth if $f$ is $C^{k}$ for all $k \in \mathbb{N}$. If we want to stress $\mathbb{K}$, we shall also write $C_{\mathbb{K}}^{k}$ in place of $C^{k}$.

In this article, we are only interested in the case where $(\mathbb{K},||$.$) is a com-$ plete valued field and both $E$ and $F$ are finite-dimensional. In the usual way, the above concept of $C^{k}$-map then gives rise to a notion of (finitedimensional) $C^{k}$-manifold and a notion of (finite-dimensional) $C^{k}$-Lie group: this is a group, equipped with a $C^{k}$-manifold structure which turns group multiplication and inversion into $C^{k}$-maps. We let $L(G):=T_{1}(G)$ denote the tangent space at the identity element $1 \in G$ and set $L(f):=T_{1}(f)$ for a $C^{k}$-homomorphism $f: G \rightarrow H$ between $C^{k}$-Lie groups. If $k \geq 3$, then the Lie bracket of left invariant vector fields can be used in the usual way to turn $L(G)$ into a Lie algebra, and $L(f)$ then is a Lie algebra homomorphism.

We mention that the $C^{k}$-maps used in this article generalize the $C^{k}$-functions of a single variable common in non-archimedian analysis (as in [26]). If $(\mathbb{K},||$.$) is a complete valued field, then a map between open subsets of finite-$ dimensional vector spaces is $C^{1}$ if and only if it is strictly differentiable at each point of its domain, in the sense of [3, 1.2.2] (see [13, Appendix C]; for locally compact fields, cf. also [11, §4]) 3 In particular, such a map is totally differentiable at each point. For a survey of differential calculus over topological fields covering various aspects of relevance for the current article, the reader is referred to [12].

Because inverse- and implicit function theorems are available for $C^{k}$-maps between finite-dimensional vector spaces over complete valued fields (see [13, notably Appendix C]) 4 we can define immersions as in the analytic case [27],

\footnotetext{
${ }^{3}$ This fact enables us to use (and cite) various results concerning $C^{1}$-maps and $C^{1}$-Lie groups from [1], 13] and 10] also in the case of non-locally compact, complete ultrametric fields, which (strictly speaking) are formulated there only in the locally compact case. The proofs only use strict differentiability and therefore carry over without changes.

${ }^{4}$ See also 11 for the cases where $k \geq 2$ or the ground field is locally compact.
} 
with analogous properties. If $k \in \mathbb{N} \cup\{\infty, \omega\}, M$ is a $C^{k}$-manifold and $N \subseteq M$ a $C^{k}$-manifold such that the inclusion map $\iota: N \rightarrow M$ is an immersion, we call $N$ an immersed $C^{k}$-submanifold of $M$; if $\iota$ is furthermore a homeomorphism onto its image, we call $N$ a $C^{k}$-submanifold. Locally around each of its points, $N$ then looks like a vector subspace inside the modelling space of $M$. Given a $C^{k}$-Lie group $G$, a subgroup $H$ equipped with a $C^{k}$-Lie group structure making it a $C^{k}$-submanifold of $G$ (resp., an immersed $C^{k}$-submanifold) is called a $C^{k}$-Lie subgroup (resp., an immersed $C^{k}$-Lie subgroup). In particular, every $C^{k}$-Lie subgroup of $G$ is closed in $G$.

1.3 Automorphisms and contraction groups. Given an automorphism $\alpha$ of a topological group $G$ and a subset $X \subseteq G$, we say that $X$ is $\alpha$-stable (resp., $\alpha$-invariant) if $\alpha(X)=X$ (resp., $\alpha(X) \subseteq X$ ). If we speak of $C^{k}$ isomorphisms between $C^{k}$-Lie groups or $C^{k}$-automorphism, we assume that the inverse map is $C^{k}$ as well. A topological group (resp., $C^{k}$-Lie group) $G$ is called contractible if it admits a contractive automorphism (resp., a contractive $C^{k}$-automorphism). Given a contraction group $(G, \alpha)$, a series $\mathbf{1}=G_{0} \triangleleft G_{1} \triangleleft \cdots \triangleleft G_{n}=G$ of $\alpha$-stable, closed subgroups of $G$ is called an $\langle\alpha\rangle$-series; it is called a composition series if it does not admit a proper refinement (see [18]).

Definition 1.4 Let $(G, \alpha)$ be a contraction group.

(a) $\alpha$ is uniformly contractive (or a uniform contraction) if each identity neighbourhood of $G$ contains an $\alpha$-invariant identity neighbourhood.

(b) $\alpha$ is compactly contractive if, for each compact set $K \subseteq G$ and identity neighbourhood $U \subseteq G$, there is $n_{0} \in \mathbb{N}$ with $\alpha^{n}(K) \subseteq U$ for all $n \geq n_{0}$.

A simple compactness argument shows that each uniformly contractive automorphism is compactly contractive.

Although our main concern are contractive automorphisms of Lie groups over local fields, some of our results will also apply to Lie groups over non-locally compact, complete ultrametric fields, like $\mathbb{C}_{p}$ and $\mathbb{Q}((X))$.

Consider a (finite-dimensional) $C^{k}$-Lie group $G$ over a complete ultrametric field $\mathbb{K}$. Then $G$ is complete (see [10, Proposition 2.1 (a)] ${ }_{5}^{5}$ and metrizable.

\footnotetext{
${ }^{5}$ Recalling footnote 3 if $k=1$ and $\mathbb{K}$ fails to be locally compact.
} 
This implies that, automatically, each contractive (bicontinuous) automorphism $\alpha$ of such a Lie group is uniformly and compactly contractive (cf. [29, Lemma 1]). Since $G$ has arbitrarily small open subgroups (see [10, Proposition 2.1]), Siebert's construction in [29] even produces small $\alpha$-invariant identity neighbourhoods.

We recall another useful fact, the proof of which exploits that contractive automorphisms of Lie groups are uniformly contractive.

1.5 Let $(\mathbb{K},||$.$) be a complete ultrametric field, G$ be a $C^{1}$-Lie group and $\alpha: G \rightarrow G$ be a contractive $C^{1}$-automorphism. Then $\beta:=L(\alpha)$ is a contractive automorphism of $L(G)$ and all eigenvalues of $\beta$ in $\overline{\mathbb{K}}$ have absolute value $<1$ (see [14]). Furthermore, there exists an ultrametric norm $\|$.$\| on \mathfrak{g}$ such that $\|\beta\|_{\text {op }}<1$ holds for the corresponding operator norm (see [14]; cf. also [8, Lemma 3.3 and its proof] and [23, Chapter II, §1]).

\section{Proof of Theorem A}

Given a contractive automorphism $\alpha$ of a totally disconnected, locally compact group $G$, there exists an $\alpha$-invariant, compact open subgroup $U$ such that $\alpha(U) \triangleleft U$ (see [28, 3.1 and Lemma 3.2]), whence $\left(\alpha^{n}(U)\right)_{n \in \mathbb{Z}}$ is a filtration for $G$ adapted to $\alpha$ in the sense of [28, 3.3]. This filtration can be used to compare structures on $G$ (or its subgroups).6 We shall use it in the proof of Theorem A to see that the $\mathbb{K}$-Lie group structure on $G$ and the $p$-adic Lie group structure on a certain hypothetical subgroup are incompatible. The next lemma will be used to relate the groups $\alpha^{n}(U)$ to balls in a local chart.

Lemma 2.1 Let $(\mathbb{K},||$.$) be a complete ultrametric field, k \in \mathbb{N} \cup\{\infty, \omega\}, G$ be a $C^{k}$-Lie group over $\mathbb{K}, S \subseteq G$ be an open subgroup and $\alpha: S \rightarrow G$ be a $C^{1}$-homomorphism. We set $\mathfrak{g}:=L(G), \beta:=L(\alpha)$ and assume that $\beta$ is a linear isomorphism and $\Theta:=\|\beta\|_{\mathrm{op}}<1$ for some ultrametric norm $\|$. on $\mathfrak{g}$. Then there exists an $\alpha$-invariant open identity neighbourhood $U \subseteq S$ and a $C^{k}$-diffeomorphism $\phi: U \rightarrow B_{r} \subseteq \mathfrak{g}$ for some $r>0$ with $\phi(1)=0$ and $T_{1}(\phi)=\mathrm{id}_{\mathfrak{g}}$, such that the sets $U_{s}:=\phi^{-1}\left(B_{s}\right)$ have the following properties:

(a) $U_{\theta s} \subseteq \alpha\left(U_{s}\right) \subseteq U_{\Theta s}$ for each $\left.\left.s \in\right] 0, r\right]$, where $\theta:=1 /\left\|\beta^{-1}\right\|_{\mathrm{op}}$.

\footnotetext{
${ }^{6}$ This idea is also the basis for the results in $[9]$.
} 
(b) $U_{s}$ is a subgroup of $G$ for each $\left.\left.s \in\right] 0, r\right]$, and a normal subgroup of $U_{r}$. Also, $U_{s} / U_{\theta s}$ is abelian for each $\left.\left.s \in\right] 0, r\right]$, and thus $\alpha\left(U_{s}\right) \triangleleft U_{s}$.

If $\operatorname{char}(\mathbb{K})=0$ and $|p|<1$ for a prime $p$, one can also achieve:

(c) $\left(U_{s}\right)^{p}=U_{|p| s}$ holds for the set of $p$-th powers, for each $\left.\left.s \in\right] 0, r\right]$.

If $\mathbb{K}$ has characteristic $p>0$, one can also achieve:

(d) For each $\varepsilon \in] 0,1\left[\right.$, there exists $\left.\left.r_{0} \in\right] 0, r\right]$ such that $\left(U_{s}\right)^{p} \subseteq U_{\varepsilon s}$ for each $\left.s \in] 0, r_{0}\right]$.

Proof. Set $\theta:=\left\|\beta^{-1}\right\|_{\text {op }}^{-1}$. Then $0<\theta<\Theta<1$. There exists a chart $\phi: U \rightarrow B_{r}$ for some open identity neighbourhood $U \subseteq S$ and some $r>0$, such that $\phi(1)=0$ and $T_{1}(\phi)=\mathrm{id}_{\mathfrak{g}}$. After shrinking $r$, we may assume that $U_{s}:=\phi^{-1}\left(B_{s}\right)$ is a subgroup of $G$ for each $\left.\left.s \in\right] 0, r\right]$, and that the remainder of (b) as well as (c) resp. (d) hold (see [10, Proposition 2.1 (b), (f) and (i)]). $]^{7}$ There exists $\left.\left.t \in\right] 0, r\right]$ such that $\alpha\left(U_{t}\right) \subseteq U_{r}$. We can therefore define a $C^{k}$-map $\gamma: B_{t} \rightarrow B_{r}$ via $\gamma(x):=\phi\left(\alpha\left(\phi^{-1}(x)\right)\right)$. Our hypotheses ensure that $\gamma$ is strictly differentiable at 0 . Now $\gamma^{\prime}(0)$ being invertible, the Ultrametric Inverse Function Theorem [11, Proposition 7.1] shows that, after shrinking $t$ if necessary, we have $\gamma\left(B_{s}\right)=\gamma^{\prime}(0)$. $B_{s}$ for each $\left.\left.s \in\right] 0, t\right]$. Since $B_{\theta s} \subseteq \gamma^{\prime}(0) . B_{s} \subseteq B_{\Theta s}$, we deduce that (a) holds after replacing $r$ with $t$.

Proof of Theorem A. We recall from [18, Theorem B] that $G=D \times T$ internally as a topological group, where $T$ is the subgroup of all torsion elements and $D$ the subgroup of all divisible elements. Also by [18, Theorem B], $D$ is a direct product $D_{p_{1}} \times \cdots \times D_{p_{m}}$, where $p_{k}$ is a prime and $D_{p_{k}}$ a nondiscrete $p_{k}$-adic Lie group for $k \in\{1, \ldots, m\}$. Let $p$ be the characteristic of $\mathbb{K}$. Then $G$ is locally pro- $p$, i.e., it has a compact open subgroup which is a pro- $p$-group (see [10, Proposition $2.1(\mathrm{~h})]$ ). Hence also each $D_{p_{k}}$ is locally pro- $p$ and hence $p_{k}=p$ (cf. [7, $\left.\S 1.2\right]$ ), whence $D$ actually is a $p$-adic Lie group. To see that $D=\{1\}$, let us assume that $D \neq\{1\}$ and derive a contradiction. Being a non-trivial contraction group, $D$ is then non-discrete (see [28, $1.8(\mathrm{c})]$ ).

Throughout the remainder of the proof, the letters (a)-(d) refer to the conditions formulated in Lemma 2.1. Applying Lemma 2.1] to $G$ and $\alpha$ (which

\footnotetext{
${ }^{7}$ The formula $\left(U_{s}\right)^{p}=U_{|p| s}$ is shown in [10] only if $|p|=p^{-1}$, but the proof works as well for arbitrary $|p|>0$.
} 
is possible by 1.5), we obtain $\left.r>0, \theta:=1 /\left\|L(\alpha)^{-1}\right\|_{\text {op }} \in\right] 0,1[$ and an $\alpha$-invariant compact open subgroup $U=U_{r} \subseteq G$ satisfying conditions (a) and (d). Then

$$
\left.\left.U_{\theta^{k} s} \subseteq \alpha^{k}\left(U_{s}\right) \text { for all } s \in\right] 0, r\right] \text { and } k \in \mathbb{N},
$$

by a simple induction based on (a). By (d), after shrinking $r$, we have

$$
U^{p^{k}} \subseteq U_{\theta^{k} r} \text { for each } k \in \mathbb{N} .
$$

Since $\left.\alpha\right|_{D}$ is a continuous (and hence analytic) contractive automorphism of the $p$-adic Lie group $D$, applying Lemma 2.1 to $\left(\mathbb{Q}_{p},|\cdot|_{p}\right), D$ and $\left.\alpha\right|_{D}$ we get some $\left.R>0, \Theta:=\left\|L\left(\left.\alpha\right|_{D}\right)\right\|_{\text {op }} \in\right] 0,1[$, a compact open subgroup $V=V_{R} \subseteq D$ and subgroups $V_{s} \subseteq V_{R}$ satisfying analogues of (a) and (c). After shrinking $R$, we may assume that $V \subseteq U$. Since $\left.\alpha\right|_{D}$ is compactly contractive, there exists $N \in \mathbb{N}$ such that $\alpha^{N}(U \cap D) \subseteq V$. Choose $\ell \in \mathbb{N}$ so large that $\ell \log _{p}(\Theta)<-1$ and set $\varepsilon:=\theta^{\ell}$. Since $U$ satisfies (d), there exists $\left.\left.r_{0} \in\right] 0, r\right]$ such that

$$
\left.\left.\left(U_{s}\right)^{p} \subseteq U_{\varepsilon s}=U_{\theta^{\ell} s} \quad \text { for each } s \in\right] 0, r_{0}\right] .
$$

There is $M \in \mathbb{N}$ such that $\theta^{M} r<r_{0}$ and hence $U^{p^{M}} \subseteq U_{\theta^{M}} \subseteq U_{r_{0}}$, using (4). Then $U^{p^{k+M}} \subseteq U_{\theta^{k \ell} r_{0}}$ for each $k \in \mathbb{N}$, by a trivial induction. Here $U_{\theta^{k} r_{0}} \subseteq$ $\alpha^{k \ell}\left(U_{r_{0}}\right)$, by (3). Thus

$$
\begin{aligned}
V_{p^{-k-M} R} & =V^{p^{k+M}} \subseteq U^{p^{k+M}} \cap D \subseteq \alpha^{k \ell}\left(U_{r_{0}}\right) \cap D \\
& \subseteq \alpha^{k \ell}(U) \cap D=\alpha^{k \ell}(U \cap D) \subseteq \alpha^{k \ell-N}(V) \subseteq V_{\Theta^{k \ell-N} R}
\end{aligned}
$$

for $k \in \mathbb{N}$ such that $k \ell \geq N$, using (c) to obtain the first equality. As a consequence, $p^{-k-M} R \leq p \Theta^{k \ell-N} R$ and hence

$$
-k-M \leq 1+(k \ell-N) \log _{p}(\Theta) \text {. }
$$

Dividing both sides of (5) by $k$ and letting $k \rightarrow \infty$, we obtain the contradiction $-1 \leq \ell \log _{p}(\Theta)$. Hence $D=\{1\}$ and thus $G=T$ is a torsion group.

We now pick a composition series (11) of $\alpha$-stable closed subgroups of $G$ (as provided by [18, Theorem 3.3]). Since $G_{j} / G_{j-1}$ is a torsion group for each $j \in\{1, \ldots, n\}$, the classification of the simple contraction groups [18, Theorem A] shows that $G_{j} / G_{j-1} \cong F_{j}^{(-\mathbb{N})} \times F_{j}^{\mathbb{N} 0}$ with the right shift, for some finite simple group $F_{j}$. Since $G$ is locally pro- $p$, so is $G_{j} / G_{j-1}$. Hence $F_{j}$ has to be a $p$-group, entailing that $F_{j} \cong C_{p}$. As a consequence, $x^{p^{n}}=1$ for each $x \in G$. Each factor $G_{j} / G_{j-1}$ being abelian, $G$ is solvable. 


\section{Contractible Lie algebras and $\mathbb{N}$-gradations}

Let us call a Lie algebra $\mathfrak{g}$ over a local field $\mathbb{K}$ contractible if there exists a contractive Lie algebra automorphism $\alpha: \mathfrak{g} \rightarrow \mathfrak{g}$. In this section, we prove the following result:

Proposition 3.1 A Lie algebra $\mathfrak{g}$ over a local field $\mathbb{K}$ is contractible if and only if it admits an $\mathbb{N}$-gradation.

The proof is based on some facts concerning automorphisms of vector spaces over ultrametric fields, which we now recall (and which will be re-used later).

3.2 Let $E$ be a finite-dimensional vector space over a complete ultrametric field $(\mathbb{K},||$.$) and \alpha$ be a linear automorphism of $E$. For each $r>0$, we let $F_{r}$ be the sum of all generalized eigenspaces of $\alpha \otimes_{\mathbb{K}} \mathrm{id}_{\overline{\mathbb{K}}}$ in $E \otimes_{\mathbb{K}} \overline{\mathbb{K}}$ to eigenvalues $\lambda \in \overline{\mathbb{K}}$ of absolute value $|\lambda|=r$. By [23, Chapter II, $\S 1], F_{r}$ is defined over $\mathbb{K}$, whence $F_{r}=E_{r} \otimes_{\mathbb{K}} \overline{\mathbb{K}}$ with $E_{r}:=F_{r} \cap E$. Then

$$
E=\bigoplus_{r>0} E_{r}
$$

We call $r \in] 0, \infty\left[\right.$ a characteristic value of $\alpha$ if $E_{r} \neq\{0\}$, and let $R(\alpha)$ be the set of characteristic values. There exists an ultrametric norm on $E$ such that

$$
\|\alpha(v)\|=r\|v\| \quad \text { for each } r \in R(\alpha) \text { and } v \in E_{r}
$$

(see [14]; cf. [8, Lemma 3.3 and its proof]). Hence $\alpha$ is contractive if and only if $R(\alpha) \subseteq] 0,1[$.

Proof of Proposition 3.1. Given a contractive Lie algebra automorphism $\alpha: \mathfrak{g} \rightarrow \mathfrak{g}$, let $\overline{\mathbb{K}}$ and $|$.$| be as in 1.1. There is a>1$ such that $\left|\mathbb{K}^{\times}\right|=\langle a\rangle \leq \mathbb{R}^{\times}$ (cf. [31] or [26, Corollary 12.2]). If $z \in \overline{\mathbb{K}}^{\times}, \mathbb{L}:=\mathbb{K}(z)$ and $d:=[\mathbb{L}: \mathbb{K}]$ is the degree of the field extension, then $|z|=\sqrt[d]{\left|N_{\mathbb{L} / \mathbb{K}}(z)\right|} \in\langle\sqrt[d]{a}\rangle$ using the norm $N_{\mathbb{L} / \mathbb{K}}(z)$ (see [21, Theorem 9.8]). Therefore

$$
\log _{a}\left|\overline{\mathbb{K}}^{\times}\right| \leq \mathbb{Q} \text {. }
$$

Applying the considerations from 3.2 to $E:=\mathfrak{g}$ and $\alpha$, we obtain $R(\alpha)$, spaces $F_{r}$ and vector subspaces $E_{r} \subseteq \mathfrak{g}$ with $\mathfrak{g}=\bigoplus_{r>0} E_{r}$. Since $R(\alpha) \subseteq$ ]0,1[, using (8) , we find $m \in \mathbb{N}$ such that that $-m \log _{a}(R(\alpha)) \subseteq \mathbb{N}$. Hence

$$
\mathfrak{g}=\bigoplus_{n \in \mathbb{N}} \mathfrak{g}_{n}
$$


with $\mathfrak{g}_{n}:=E_{a^{-n / m}}$. Since $\left[F_{r}, F_{s}\right] \subseteq F_{r s}$ and hence $\left[E_{r}, E_{s}\right] \subseteq E_{r s}$ for all $r, s>0$ as a consequence of Proposition 12 (i) in [5, Chapter 7, §1, no. 4], it follows that (9) is an $\mathbb{N}$-gradation.

Conversely, assume that $\mathfrak{g}=\bigoplus_{n \in \mathbb{N}} \mathfrak{g}_{n}$ is an $\mathbb{N}$-gradation. Pick $\theta \in \mathbb{K}^{\times}$such that $|\theta|<1$. Then the unique $\mathbb{K}$-linear map $\alpha: \mathfrak{g} \rightarrow \mathfrak{g}$ taking $x \in \mathfrak{g}_{n}$ to $\theta^{n} x$ is a contractive Lie algebra automorphism of $\mathfrak{g}$.

\section{Contractible Lie groups are nilpotent}

In this section, we prove Theorem B. The proof uses the stable manifolds for ultrametric dynamical systems constructed in [14] by an adaptation of Irwin's method (as in [20] and [32]) 8

4.1 Let $(\mathbb{K},||$.$) be a complete ultrametric field and k \in \mathbb{N} \cup\{\infty, \omega\}$. Let $M$ be a finite-dimensional $C^{k}$-manifold over $\mathbb{K}, \alpha: M \rightarrow M$ be a $C^{k}$-diffeomorphism and $z \in M$ be a fixed point of $\alpha$. Write $r_{1}<\cdots<r_{n}$ for the characteristic values of $T_{z}(\alpha)$. Given $\left.a \in\right] 0,1\left[\backslash\left\{r_{1}, \ldots, r_{n}\right\}\right.$, let $W_{a}^{s}(M, z)$ be the set of all $x \in M$ with the following property: For some (and hence each) chart $\phi: U \rightarrow V \subseteq T_{z}(M)$ of $M$ around $z$ such that $\phi(z)=0$ and $T_{z}(\phi)=\operatorname{id}_{T_{z}(M)}$, and some (and hence each) norm $\|$.$\| on T_{z}(M)$, there exists $n_{0} \in \mathbb{N}$ such that $\alpha^{n}(x) \in U$ for all integers $n \geq n_{0}$ and

$$
\lim _{n \rightarrow \infty} \frac{\left\|\phi\left(\alpha^{n}(x)\right)\right\|}{a^{n}}=0 .
$$

It is clear from the definition that $W_{a}^{s}(M, z)$ is an $\alpha$-stable subset of $M$. The following facts are proved in [14]:

4.2 For each $a \in] 0,1\left[\backslash\left\{r_{1}, \ldots, r_{n}\right\}\right.$, the set $W_{a}^{s}(M, z)$ is an immersed $C^{k}$ submanifold of $M$. It is called the a-stable manifold of $M$ around $z$.

4.3 If $\left.\left.\left\{r_{1}, \ldots, r_{n}\right\} \subseteq\right] 0,1\right]$, then $W_{a}^{s}(M, z)$ is a $C^{k}$-submanifold of $M$, for each $a \in] 0,1\left[\backslash\left\{r_{1}, \ldots, r_{n}\right\}\right.$.

4.4 If $0<a<b<1$ and $[a, b] \cap\left\{r_{1}, \ldots, r_{n}\right\}=\emptyset$, then $W_{a}^{s}(M, z)=W_{b}^{s}(M, z)$.

4.5 If $a \in] 0, r_{1}\left[\right.$, then $W_{a}^{s}(M, z)=\{z\}$.

\footnotetext{
${ }^{8}$ See also [1] and [12 for outlines of the main steps of this construction.
} 
Proposition 4.6 Let $k \in \mathbb{N} \cup\{\infty, \omega\}$ and $(\mathbb{K},||$.$) be a complete ultrametric$ field. Let $G$ be a $C^{k}$-Lie group over $\mathbb{K}$ and $\alpha: G \rightarrow G$ be a $C^{k}$-automorphism. Assume that $a \in] 0,1[$ is not a characteristic value of $L(\alpha)$. Then the a-stable manifold $W_{a}^{s}(G, 1)$ is an immersed $C_{\mathbb{K}}^{k}$-Lie subgroup of $G$. If $\alpha$ is a contractive $C^{k}$-automorphism, then $W_{a}^{s}(G, 1)$ is a $C_{\mathbb{K}^{-}}^{k}$ Lie subgroup of $G$.

Proof. We first show that $H:=W_{a}^{s}(G, 1)$ is a subgroup of $G$. To this end, we pick a chart $\phi: U \rightarrow V \subseteq T_{1}(G)=L(G)$ as in 4.1 and an ultrametric norm $\|$.$\| on L(G)$; we use the same symbol, $\|$.$\| , for the corresponding max-$ imum norm on $L(G) \times L(G)$. After shrinking $U$, we may assume that $U$ is a subgroup of $G$ and give $V$ the group structure making $\phi$ a homomorphism (see [10, Proposition 2.1]). After shrinking $U$ further, we may assume that

$$
\left\|x y^{-1}-(x-y)\right\| \leq\|(x, y)\| \text { for all } x, y \in V,
$$

because $h: V \times V \rightarrow V,(x, y) \mapsto x y^{-1}$ is totally differentiable at $(0,0)$ with $h^{\prime}(0,0): L(G) \times L(G) \rightarrow L(G),(u, v) \mapsto u-v$.

If $x, y \in H$, there exists $n_{0} \in \mathbb{N}$ such that $\alpha^{n}(x), \alpha^{n}(y) \in U$ for all $n \geq n_{0}$ and $\left\|\phi\left(\alpha^{n}(x)\right)\right\| / a^{n},\left\|\phi\left(\alpha^{n}(y)\right)\right\| / a^{n} \rightarrow 0$. Then $\alpha^{n}\left(x y^{-1}\right)=\alpha^{n}(x) \alpha^{n}(y)^{-1} \in$ $U U^{-1}=U$ and

$$
\begin{aligned}
& \left\|\phi\left(\alpha^{n}\left(x y^{-1}\right)\right)\right\| / a^{n}=\left\|\phi\left(\alpha^{n}(x)\right) \phi\left(\alpha^{n}(y)\right)^{-1}\right\| / a^{n} \\
& \quad \leq \max \left\{\left\|\phi\left(\alpha^{n}(x)\right)-\phi\left(\alpha^{n}(y)\right)\right\|,\left\|\left(\phi\left(\alpha^{n}(x)\right), \phi\left(\alpha^{n}(y)\right)^{-1}\right)\right\|\right\} / a^{n} \\
& \quad=\max \left\{\left\|\phi\left(\alpha^{n}(x)\right)\right\| / a^{n},\left\|\phi\left(\alpha^{n}(y)\right)\right\| / a^{n}\right\} \rightarrow 0
\end{aligned}
$$

as $n \rightarrow \infty$, showing that $x y^{-1} \in H$. Hence $H$ is a subgroup indeed.

To see that $H$ is an immersed $C^{k}$-Lie subgroup, we recall from the construction of $a$-stable manifolds that $\left.\alpha\right|_{H}: H \rightarrow H$ is a $C^{k}$-diffeomorphism and that there is an $\alpha$-invariant open subset $\Gamma \subseteq H$ (a "local $a$-stable manifold") such that $H=\bigcup_{n \in \mathbb{N}_{0}} \alpha^{-n}(\Gamma)$ and $\Gamma$ is a submanifold of $G$. For a suitable choice of the chart $\phi: U \rightarrow V$ and ultrametric norm $\|$.$\| on L(G)$ above, one has $V=B_{r} \subseteq L(G)$ for some $r>0$ in the construction and the set $\Gamma$ consists of all $x \in U$ such that

$(\diamond) \alpha^{n}(x) \in U$ for all $n \in \mathbb{N}_{0},\left\|\phi\left(\alpha^{n}(x)\right)\right\| \leq a^{n}$ for all $n \in \mathbb{N}_{0}$, and $\left\|\phi\left(\alpha^{n}(x)\right)\right\| / a^{n} \rightarrow 0$ as $n \rightarrow \infty$

(see [14]). After shrinking $U$ (and $r$ ) if necessary, we may assume that $U$ is a subgroup of $G$ and the estimates (11) hold. Given $x, y \in \Gamma$, we can use 
(11) as above to see that also $x y^{-1}$ satisfies the conditions $(\diamond)$, and hence $x y^{-1} \in \Gamma$. Thus $\Gamma$ is a subgroup of $G$ and hence a $C^{k}$-Lie subgroup. As a consequence, also $\alpha^{-n}(\Gamma)$ is a $C^{k}$-Lie subgroup of $G$. Since each $\alpha^{-n}(\Gamma)$ is an open $C^{k}$-submanifold of the $a$-stable manifold $H$ and $H=\bigcup_{n \in \mathbb{N}_{0}} \alpha^{-n}(\Gamma)$, it follows that the group operations of $H$ are $C^{k}$ on an open cover and hence $C^{k}$. Thus $H$ is an immersed $C^{k}$-Lie subgroup of $G$.

If $\alpha$ is contractive, then $R(\alpha) \subseteq] 0,1\left[\right.$ (see 1.5). Hence $H=W_{a}^{s}(G, 1)$ is a $C^{k}$-submanifold of $G$ (by 4.3 ) and therefore a $C^{k}$-Lie subgroup.

Given subsets $X, Y$ of a group $G$, we set $[X, Y]:=\left\{x y x^{-1} y^{-1}: x \in X, y \in Y\right\}$.

Lemma 4.7 Let $G$ be a $C^{2}$-Lie group over a complete ultrametric field $(\mathbb{K},||$.$) . Let \alpha: G \rightarrow G$ be a $C^{2}$-automorphism and assume that $\left.a, b \in\right] 0,1[$ as well as ab are not characteristic values of $L(\alpha)$. Then

$$
\left[W_{a}^{s}(G, 1), W_{b}^{s}(G, 1)\right] \subseteq W_{a b}^{s}(G, 1) .
$$

Proof. We pick a chart $\phi: U \rightarrow V \subseteq L(G)$ of $G$ around 1 such that $\phi(1)=0$ and $T_{1}(\phi)=\operatorname{id}_{L(G)}$. After shrinking $U$ further, we may assume that $U$ is a subgroup of $G$. We give $V$ the group structure making $\phi$ an isomorphism. Then $V$ is a $C^{2}$-Lie group. The commutator map $f: V \times V \rightarrow V$, $f(x, y)=x y x^{-1} y^{-1}$ is $C^{2}$ and satisfies $f(x, 0)=f(0, y)=0$. Hence, by [10, Lemma 1.7], after shrinking $V$ there exists $C>0$ such that

$$
\|f(x, y)\| \leq C\|x\| \cdot\|y\| \quad \text { for all } x, y \in V .
$$

Given $x \in W_{a}^{s}(G, 1)$ and $y \in W_{b}^{s}(G, 1)$, there exists $n_{0} \in \mathbb{N}$ such that $\alpha^{n}(x), \alpha^{n}(y) \in U$ for all $n \geq n_{0}$. Then

$$
\begin{aligned}
\frac{\left\|\phi\left(\alpha^{n}\left(x y x^{-1} y^{-1}\right)\right)\right\|}{(a b)^{n}} & =\frac{\left\|f\left(\phi\left(\alpha^{n}(x)\right), \phi\left(\alpha^{n}(y)\right)\right)\right\|}{(a b)^{n}} \\
& \leq C \frac{\left\|\phi\left(\alpha^{n}(x)\right)\right\|}{a^{n}} \frac{\left\|\phi\left(\alpha^{n}(y)\right)\right\|}{b^{n}} \rightarrow 0
\end{aligned}
$$

as $n \rightarrow \infty$ (see (10)), and thus $x y x^{-1} y^{-1} \in W_{a b}^{s}(G, 1)$.

Proof of Theorem B. We may assume that $G \neq\{1\}$. Since $\alpha$ is contractive, it follows that $R(L(\alpha)) \subseteq] 0,1\left[\right.$ (see 1.5). Let $0<r_{1}<\cdots<r_{m}<1$ be the 
characteristic values of $L(\alpha)$. Pick $\left.a_{m} \in\right] r_{m}, 1[$. Next, for $j \in\{1, \ldots, m-1\}$, pick $\left.a_{j} \in\right] r_{j}, r_{j+1}\left[\right.$ so small that $a_{j} a_{m}<r_{j}$, and such that $a_{j} a_{i} \notin\left\{r_{1}, \ldots, r_{m}\right\}$ for all $i \geq j$. Set $a_{0}:=a_{1} a_{n}$. By Proposition 4.6, $G_{j}:=W_{a_{j}}^{s}(G, 1)$ is a $C^{k}$-Lie subgroup of $G$, for $j \in\{0,1, \ldots, m\}$. Furthermore, each $G_{j}$ is $\alpha$-stable, and $G_{0}=\{1\}$, by 4.5. Also, $G_{m}=G$ (cf. [14]). By Lemma 4.7, we have

$$
\left[G, G_{j}\right]=\left[W_{a_{m}}^{s}(G, 1), W_{a_{j}}^{s}(G, 1)\right] \subseteq W_{a_{m} a_{j}}^{s}(G, 1) \subseteq W_{a_{j-1}}^{s}(G, 1)=G_{j-1}
$$

for $j \in\{1, \ldots, m\}$. Hence each $G_{j}$ is normal in $G$ and $G_{j} / G_{j-1}$ is contained in the centre of $G / G_{j-1}$, showing that $\mathbf{1}=G_{0} \triangleleft G_{1} \triangleleft \cdots \triangleleft G_{m}=G$ is a central series. In particular, $G$ is nilpotent (see [25, p. 122]).

If $\alpha$ merely is a contractive $C^{1}$-automorphism in Theorem $\mathrm{B}$, the preceding proof still provides a central series of $C^{1}$-Lie subgroups (it is only essential that the commutator map $f$ is $C^{2}$ ).

\section{From contractible Lie algebras to contractible Lie groups}

In this section, we discuss the passage from contractive Lie algebra automorphisms to contractive Lie group automorphisms. We begin with a result which subsumes Theorem $\mathrm{C}$ from the introduction (when specialized to characteristic 0). Afterwards, we work towards a category-theoretic refinement of Theorem C: an equivalence between the category of analytic Lie contraction groups and the category of Lie algebra-contraction pairs.

Proposition 5.1 Let $(\mathbb{K},||$.$) be a complete ultrametric field, k \in \mathbb{N} \cup\{\infty, \omega\}$ such that $k \geq 3, \mathfrak{g}$ be a Lie algebra over $\mathbb{K}$ and $\beta: \mathfrak{g} \rightarrow \mathfrak{g}$ be a contractive Lie algebra automorphism. We make the following assumption:

(*) There exists a $C^{k}$-Lie group $V$ with $L(V)=\mathfrak{g}$, an open subgroup $U \subseteq V$ and a $C^{k}$-homomorphism $\gamma: U \rightarrow V$ such that $L(\gamma)=\beta$.

Then there exists a $C^{k}$-Lie group $G$ and a contractive $C^{k}$-automorphism $\alpha: G \rightarrow G$ such that $L(\alpha)=\beta$. If $\operatorname{char}(\mathbb{K})=0$, then condition $(*)$ is automatically satisfied. If char $(\mathbb{K})=0$ and $k=\omega$, then furthermore $(G, \alpha)$ is unique up to isomorphism.

Proof. By 3.2, there exists an ultrametric norm $\|$.$\| on \mathfrak{g}$ such that $\Theta:=$ $\|\beta\|_{\text {op }}<1$. Hence Lemma 2.1 applies to $\gamma: U \rightarrow V$, and ensures that after 
shrinking $U$, there is $r>0$ and a chart $\phi: U \rightarrow B_{r} \subseteq \mathfrak{g}$ with $\phi(1)=0$ and $T_{1}(\phi)=\mathrm{id}_{\mathfrak{g}}$ such that $\gamma\left(U_{s}\right) \subseteq U_{\Theta s}$ for each $\left.\left.s \in\right] 0, r\right]$, with $U_{s}:=\phi^{-1}\left(B_{s}\right)$. In particular, this implies that $\gamma(U) \subseteq U$ and that $\left\{\gamma^{n}(U): n \in \mathbb{N}_{0}\right\}$ is a basis of $\gamma$-stable identity neighbourhoods in $U$ ( since $\gamma^{n}(U) \subseteq U_{\Theta^{n} r}$ ). Hence $\left.\gamma\right|_{U}: U \rightarrow U$ is contractive. Since $T_{1}(\gamma)=\beta$ is invertible, after shrinking $r$ if necessary we may assume that also $\gamma(U)$ is open in $U$ and $\gamma: U \rightarrow \gamma(U)$ is a $C^{k}$-diffeomorphism (using the Inverse Function Theorem [13, Theorem 5.1], resp., [27, Part II, Chapter III, §9, Theorem 2]).

The group $V$ together with the isomorphism $\left.\gamma\right|_{U}: U \rightarrow \gamma(U)$ between its subgroups gives rise to an HNN-extension $W$. This is a group $W$ which contains $V$ as a subgroup and has an element $w \in W$ such that

$$
w x w^{-1}=\gamma(x) \text { for all } x \in U
$$

(see, e.g., 6.4.5 and the remarks following it in [25]). Consider the inner automorphism $\alpha: W \rightarrow W, \alpha(x):=w x w^{-1}$. Then $\alpha(U) \subseteq U$ and $\left.\alpha\right|_{U}$ is $C^{k}$. Furthermore, $\left.\alpha^{-1}\right|_{\alpha(U)}$ is $C^{k}$ on the open identity neighbourhood $\alpha(U) \subseteq U$. Since $U$ is a $C^{k}$-Lie group, standard arguments now provide a unique $C^{k}$ Lie group structure on the subgroup $H:=\langle U \cup\{w\}\rangle \leq W$ generated by $U$ and $w$ which makes $U$ an open $C^{k}$-submanifold of $H$. Since $\alpha(U)=\gamma(U) \subseteq$ $U$, it follows that $G:=\bigcup_{n \in \mathbb{N}} \alpha^{-n}(U) \subseteq H$ is an open subgroup of $H$ and $\left.\alpha\right|_{G}: G \rightarrow G$ a contractive automorphism with $L\left(\left.\alpha\right|_{G}\right)=L(\gamma)=\beta$.

If $\operatorname{char}(\mathbb{K})=0$, we choose an ultrametric norm $\|$.$\| on \mathfrak{g}$ such that $\|\beta\|_{\text {op }}<1$. For some $t>0$, the Baker-Campbell-Hausdorff (BCH-) series then converges on $B_{t} \times B_{t}$ (where $B_{t}:=B_{t}^{\mathfrak{g}}(0)$ ) to a function taking its values in $B_{t}$, and making $U:=B_{t}$ a $\mathbb{K}$-analytic Lie group with $L(U)=\mathfrak{g}$ (see Lemma 3 in [4, Chapter 3, $\S 4$, no.2]). Then $V:=U$ together with $\gamma:=\left.\beta\right|_{U}$ satisfies condition $(*)$. The uniqueness assertion is covered by Lemma 5.4 below.

If $\operatorname{char}(\mathbb{K})>0$, then an analytic Lie contraction group $(G, \alpha)$ need not be determined by $(L(G), L(\alpha))$ (see Example 6.7).

Remark 5.2 The preceding proof shows that if $(*)$ holds, then after shrinking $U$ we can assume that

(**) There is a $C^{k}$-Lie group $U$ and a $C^{k}$-homomorphism $\gamma: U \rightarrow U$ with open image such that $L(\gamma)=\beta$ and $\gamma: U \rightarrow \gamma(U)$ is a $C^{k}$-diffeomorphism with $\left\{\gamma^{n}(U): n \in \mathbb{N}_{0}\right\}$ a basis of identity neighbourhoods in $U$. 
In this case, $(G, \alpha)$ can be chosen such that $U$ is an open subgroup of $G$ and $\left.\alpha\right|_{U}=\gamma$.

The following lemma is a variant of [30, Proposition 2.2].

Lemma 5.3 Let $\left(G_{1}, \alpha_{1}\right)$ be a contraction group, $U \subseteq G_{1}$ be an $\alpha_{1}$-invariant open subgroup, $G_{2}$ be a group, $\alpha_{2}$ be an automorphism of $G_{2}$ and $g: U \rightarrow G_{2}$ be a homomorphism such that $\alpha_{2} \circ g=\left.g \circ \alpha_{1}\right|_{U}$. Then $g$ extends uniquely to a homomorphism $h: G_{1} \rightarrow G_{2}$ such that $\alpha_{2} \circ h=h \circ \alpha_{1}$. If also $\left(G_{2}, \alpha_{2}\right)$ is a contraction group, $g(U)$ open and $g: U \rightarrow g(U)$ a homeomorphism, then $h$ is an isomorphism of topological groups.

Proof. First assertion: The hypotheses ensure $U \subseteq \alpha_{1}^{-1}(U) \subseteq \alpha_{1}^{-2}(U) \subseteq \cdots$ and $G_{1}=\bigcup_{n \in \mathbb{N}_{0}} \alpha_{1}^{-n}(U)$. Given $x \in \alpha_{1}^{-n}(U)$, we set $h(x):=\alpha_{2}^{-n}\left(g\left(\alpha_{1}^{n}(x)\right)\right)$. It is easy to see that $h$ is well defined and has the desired properties.

Second assertion: Since $V:=g(U)$ satisfies $\alpha_{2}(V) \subseteq V$ and $\alpha_{1} \circ g^{-1}=$ $\left.g^{-1} \circ \alpha_{2}\right|_{V}$, the first assertion yields a homomorphism $k: G_{2} \rightarrow G_{1}$ such that $\left.k\right|_{V}=g^{-1}$ and $\alpha_{1} \circ k=k \circ \alpha_{2}$. Then $h \circ k=\mathrm{id}_{G_{2}}$ and $k \circ h=\mathrm{id}_{G_{1}}$, by the uniqueness assertion.

Lemma 5.4 Let $(\mathbb{K},||$.$) be a complete ultrametric field of characteristic 0$. Let $G_{j}$ be an analytic Lie group over $\mathbb{K}$ and $\alpha_{j}$ be a contractive, analytic automorphism of $G_{j}$, for $j \in\{1,2\}$. Let $f: L\left(G_{1}\right) \rightarrow L\left(G_{2}\right)$ be a Lie algebra homomorphism with $f \circ L\left(\alpha_{1}\right)=L\left(\alpha_{2}\right) \circ f$. Then there is a unique analytic homomorphism $f^{\wedge}: G_{1} \rightarrow G_{2}$ such that $L\left(f^{\wedge}\right)=f$ and $\alpha_{2} \circ f^{\wedge}=f^{\wedge} \circ \alpha_{1}$. If $f$ is a Lie algebra isomorphism, then $f^{\wedge}$ is an analytic isomorphism.

Proof. By Lemma 2.1 and 1.5, $G_{1}$ has arbitrarily small $\alpha_{1}$-invariant open subgroups $U$. By Theorem 1 (i) in [4, Chapter 3, $\S 4$, no. 1], after choosing $U$ small enough there exists an analytic homomorphism $g: U \rightarrow G_{2}$ such that $L(g)=f$. Since $L\left(\left.g \circ \alpha_{1}\right|_{U}\right)=L(g) \circ L\left(\alpha_{1}\right)=f \circ L\left(\alpha_{1}\right)=L\left(\alpha_{2}\right) \circ f=L\left(\alpha_{2} \circ g\right)$, part (ii) of the theorem just cited shows that $\left.g \circ \alpha_{1}\right|_{U}=\alpha_{2} \circ g$, after choosing $U$ even smaller if necessary. Now Lemma 5.3 provides a unique homomorphism $f^{\wedge}: G_{1} \rightarrow G_{2}$ such that $\alpha_{2} \circ f^{\wedge}=f^{\wedge} \circ \alpha_{1}$ and $\left.f^{\wedge}\right|_{U}=g$. Since $g$ is analytic, so is $f^{\wedge}$, and $L\left(f^{\wedge}\right)=L(g)=f$.

If also $f^{*}: G_{1} \rightarrow G_{2}$ is an analytic homomorphism with the desired properties, then $\left.f^{*}\right|_{V}=\left.f^{\wedge}\right|_{V}$ for a sufficiently small $\alpha$-stable open subgroup $V \subseteq G_{1}$ 
(which a priori might be smaller than $U$ just used), because $L\left(f^{*}\right)=L\left(f^{\wedge}\right)$. Hence $f^{*}=f^{\wedge}$ by uniqueness in Lemma 5.3. To complete the proof, note that $g(U)$ is open and $g: U \rightarrow g(U)$ is an analytic diffeomorphism in the preceding construction if we choose $U$ sufficiently small, and hence $f^{\wedge}$ is an isomorphism by Lemma 5.3 .

Definition 5.5 Let $(\mathbb{K},||$.$) be a complete ultrametric field of characteristic 0$. We then obtain categories $\mathbb{C L} \mathbb{G}_{\mathbb{K}}$ and $\mathbb{C L} \mathbb{A}_{\mathbb{K}}$, as follows:

- The objects of $\mathbb{C L} \mathbb{G}_{\mathbb{K}}$ are pairs $(G, \alpha)$, where $G$ is an analytic Lie group over $\mathbb{K}$ and $\alpha: G \rightarrow G$ a contractive, analytic automorphism. A morphism $\left(G_{1}, \alpha_{1}\right) \rightarrow\left(G_{2}, \alpha_{2}\right)$ in $\mathbb{C L} \mathbb{G}_{\mathbb{K}}$ is an analytic homomorphism $f: G_{1} \rightarrow G_{2}$ such that $\alpha_{2} \circ f=f \circ \alpha_{1}$.

- The objects of $\mathbb{C L}_{\mathbb{K}}$ are pairs $(\mathfrak{g}, \beta)$, where $\mathfrak{g}$ is a Lie algebra over $\mathbb{K}$ and $\beta: \mathfrak{g} \rightarrow \mathfrak{g}$ a contractive Lie algebra automorphism. A morphism $\left(\mathfrak{g}_{1}, \beta_{1}\right) \rightarrow\left(\mathfrak{g}_{2}, \beta_{2}\right)$ is a Lie algebra homomorphism $f: \mathfrak{g}_{1} \rightarrow \mathfrak{g}_{2}$ such that $\beta_{2} \circ f=f \circ \beta_{1}$.

We now show:

Theorem 5.6 The categories $\mathbb{C L} \mathbb{G}_{\mathbb{K}}$ and $\mathbb{C L}_{\mathbb{K}}$ are equivalent.

Proof. It is clear that a covariant functor $P: \mathbb{C L} \mathbb{G}_{\mathbb{K}} \rightarrow \mathbb{C L}_{\mathbb{K}}$ can be defined via $P(G, \alpha):=(L(G), L(\alpha))$ on objects and $P(f):=L(f)$ on morphisms (cf. 1.5). We now define a covariant functor $Q: \mathbb{C L}_{\mathbb{K}} \rightarrow \mathbb{C L} \mathbb{G}_{\mathbb{K}}$. Given an object $x=(\mathfrak{g}, \beta)$, we let $Q(x):=(G, \alpha)$ be an analytic Lie contraction group such that $L(G)=\mathfrak{g}$ and $L(\alpha)=\beta$, as constructed in Proposition 5.1. More precisely, we identify $\mathfrak{g}$ with $L(G)$ here by means of a fixed Lie algebra isomorphism

$$
\phi_{x}: \mathfrak{g} \rightarrow L(G),
$$

and require that $L(\alpha)=\phi_{x} \circ \beta \circ \phi_{x}^{-1}$. Given objects $x_{j}=\left(\mathfrak{g}_{j}, \beta_{j}\right)$ for $j \in\{1,2\}$ and a morphism $f:\left(\mathfrak{g}_{1}, \beta_{1}\right) \rightarrow\left(\mathfrak{g}_{2}, \beta_{2}\right)$, we define a morphism $Q\left(x_{1}\right) \rightarrow Q\left(x_{2}\right)$ via $Q(f):=\left(\phi_{x_{2}} \circ f \circ \phi_{x_{1}}^{-1}\right)^{\wedge}$, using notation as in Lemma 5.4. Then it is easy to see that $Q$ is a functor and that $\phi$ is a natural isomorphism from id to $P \circ Q$ (in the sense of [22, p. 16]). Furthermore, a natural isomorphism $\psi$ from id to $Q \circ P$ can be defined as follows:

Given an object $y=(G, \alpha)$ in $\mathbb{C} \mathbb{L} \mathbb{G}_{\mathbb{K}}$, we have $P(y)=(L(G), L(\alpha))=: x$ and 
$Q(P(y))=\left(G_{x}, \alpha_{x}\right)$, where $L\left(\alpha_{x}\right)=\phi_{x} \circ L(\alpha) \circ \phi_{x}^{-1}$. By Lemma 5.4, there exists a unique isomorphism $\psi_{y}:=\left(\phi_{x}\right)^{\wedge}: G \rightarrow G_{x}$ such that $\alpha_{x} \circ \psi_{y}=\psi_{y} \circ \alpha$ and $L\left(\psi_{y}\right)=\phi_{x}: L(G) \rightarrow L\left(G_{x}\right)$. The naturality is easy to check.

We have shown that the functors $P$ and $Q$ define an equivalence of categories between $\mathbb{C L} \mathbb{G}_{\mathbb{K}}$ and $\mathbb{C L}_{\mathbb{K}}$ (in the sense of [22, p. 18]).

\section{Examples and open problems}

We start with examples related to Theorem A.

Example 6.1 Let $\mathbb{K}$ be a local field of positive characteristic. Since $\mathbb{K}^{\times}$ is not a torsion group, it does not admit a contractive $C^{1}$-automorphism, by Theorem A. In fact, $\mathbb{K}^{\times}$does not even admit a contractive bicontinuous automorphism. To see this, we assume the existence of such an automorphism $\alpha$ and derive a contradiction. We pick an element $0 \neq x \in \mathbb{K}^{\times}$such that $|x| \neq 1$. Then $D:=\langle x\rangle$ is an infinite cyclic group and discrete in the topology induced by $\mathbb{K}^{\times}$. We let $U \subseteq \mathbb{K}^{\times}$be a compact open subgroup. Then $\alpha^{n}(x) \in U$ for some $n$ and hence $\alpha^{n}(D)$ is an infinite discrete subgroup of the compact group $U$, which is absurd.

The following example shows that it is in general not possible to choose all of the groups $G_{j}$ in a composition series (1) as $C_{\mathbb{K}^{-}}^{k}$ Lie subgroups of $G$.

Example 6.2 Let $\mathbb{K}:=\mathbb{F}_{p}((X))$ be the field of formal Laurent series over a finite field $\mathbb{F}_{p}$ with $p$ elements, $G:=(\mathbb{K},+)$ and $\alpha: G \rightarrow G, z \mapsto X^{2} z$. Then $G_{1}:=\mathbb{F}_{p}^{(-2 \mathbb{N})} \times \mathbb{F}_{p}^{2 \mathbb{N}_{0}} \subseteq \mathbb{F}_{p}^{(-\mathbb{N})} \times \mathbb{F}_{p}^{\mathbb{N}_{0}}=\mathbb{K}$ is an $\alpha$-stable closed subgroup of $G$. Furthermore $G_{1} \cong \mathbb{F}_{p}^{(-\mathbb{N})} \times \mathbb{F}_{p}^{\mathbb{N}_{0}}$ via $\left(x_{n}\right)_{n \in 2 \mathbb{Z}} \mapsto\left(x_{2 n}\right)_{n \in \mathbb{Z}}$ and $G / G_{1} \cong$ $\mathbb{F}^{(-2 \mathbb{N}+1)} \times \mathbb{F}^{2 \mathbb{N}_{0}+1}$. As both contraction groups are isomorphic to $C_{p}^{(-\mathbb{N})} \times C_{p}^{\mathbb{N}_{0}}$ with the right shift, they are simple contraction groups and hence

$$
1 \triangleleft G_{1} \triangleleft G
$$

is a composition series of closed $\alpha$-stable subgroups of $G$. Let $\mathbf{1} \triangleleft H_{1} \triangleleft G$ be any such composition series. We now show that $H_{1}$ is not a Lie subgroup. In fact, $H_{1}$ is a non-discrete, proper subgroup of $G$. Hence, if $H_{1}$ would be a Lie subgroup of $G$, it would be 1-dimensional and hence open in the 1dimensional Lie group $G$. Then $G=\bigcup_{n \in \mathbb{N}_{0}} \alpha^{-n}\left(H_{1}\right)=H_{1}$, which is absurd. 
In other cases, the groups $G_{j}$ can be chosen only as $C_{\mathbb{L}}^{k}$-Lie subgroups for some subfield $\mathbb{L} \subseteq \mathbb{K}$.

Example 6.3 Let $\mathbb{K}:=\mathbb{F}_{p^{2}}((X)), G:=(\mathbb{K},+)$ and $\alpha: G \rightarrow G, z \mapsto X z$. Then $G_{1}:=\mathbb{F}_{p}((X))$ is an $\alpha$-stable closed subgroup $G$. Since both $G_{1}$ and $G / G_{1}$ are isomorphic as contraction groups to $C_{p}^{(-\mathbb{N})} \times C_{p}^{\mathbb{N}_{0}}$ with the right shift, they are simple contraction groups and hence $1 \triangleleft G_{1} \triangleleft G$ is a composition series of closed $\alpha$-stable subgroups of $G$. Here $G_{1}$ is a $C_{\mathbb{L}}^{\omega}$-Lie subgroup over $\mathbb{L}:=\mathbb{F}_{p}((X))$. However, neither $G_{1}$ nor any other group $H_{1}$ in a composition series $1 \triangleleft H_{1} \triangleleft G$ of $\alpha$-stable closed subgroups can be a $C_{\mathbb{K}^{-}}^{1}$ Lie subgroup, because $G$ is 1-dimensional over $\mathbb{K}$, enabling us to argue as in Example 6.2.

Of course, instead of a composition series of closed $\alpha$-stable subgroups, in the situation of Theorem A we can consider a properly ascending series

$$
\mathbf{1}=G_{0} \triangleleft G_{1} \triangleleft \cdots \triangleleft G_{m}=G
$$

of $\alpha$-stable $C^{k}$-Lie subgroups $G_{j}$ of $G$ which cannot be properly refined to a series of the same type (let us call such a series a Lie composition series). In other words, each factor $G_{j} / G_{j-1}$ is a simple Lie contraction group of class $C^{k}$ in the sense that it is non-trivial and does not have a proper, normal, non-trivial $C^{k}$-Lie subgroup stable under the contractive $C^{k}$-automorphism induced by $\alpha$.

We mention that Lie composition series also exist if $(\mathbb{K},||$.$) is a non-locally$ compact, complete ultrametric field, because $\operatorname{dim}_{\mathbb{K}}\left(G_{j}\right)<\operatorname{dim}_{\mathbb{K}}\left(G_{j+1}\right)$ holds for the groups in a strictly ascending Lie series.

As a consequence of Theorem B, every simple Lie contraction group $(G, \alpha)$ of class $C^{k}$ (with $k \geq 2$ ) over a complete ultrametric field is abelian. If $\operatorname{char}(\mathbb{K})=0$ and $k=\omega$, this easily implies that $G$ is isomorphic to $\left(\mathbb{K}^{n},+\right)$ for some $n$ and $\alpha$ corresponds to a $\mathbb{K}$-linear automorphism. If $\mathbb{K}$ has positive characteristic, then currently we cannot say more.

Problem 6.4 Is it possible to classify all simple Lie contraction groups over complete ultrametric fields of positive characteristic, or at least over local fields of positive characteristic?

The following example shows that simple Lie contraction groups need not be 1-dimensional - each given dimension $n \in \mathbb{N}$ can occur. 
Example 6.5 Let $\mathbb{K}:=\mathbb{F}_{p}((X)), G:=\left(\mathbb{K}^{2},+\right)$ and $\alpha: G \rightarrow G$ be defined via $(x, y) \mapsto(X y, x)$. Then the map $\phi: G \rightarrow \mathbb{K}=\mathbb{F}_{p}^{(-\mathbb{N})} \times \mathbb{F}_{p}^{\mathbb{N}_{0}}$,

$$
\left(\sum_{k} a_{k} X^{k}, \sum_{k} b_{k} X^{k}\right) \mapsto \sum_{k} a_{k} X^{2 k}+\sum_{k} b_{k} X^{2 k+1}
$$

is an isomorphism of topological groups and $\phi \circ \alpha \circ \phi^{-1}$ is the right shift on $\mathbb{F}_{p}^{(-\mathbb{N})} \times \mathbb{F}_{p}^{\mathbb{N}_{0}}$. Hence $(G, \alpha)$ is a simple contraction group. As a Lie group, $G$ is 2-dimensional.

Analogous arguments show that the $n$-dimensional $\mathbb{K}$-analytic Lie group $\mathbb{K}^{n}$, together with $\alpha: \mathbb{K}^{n} \rightarrow \mathbb{K}^{n}, \alpha\left(x_{1}, \ldots, x_{n}\right):=\left(X x_{n}, x_{1}, \ldots, x_{n-1}\right)$, is isomorphic to $\mathbb{F}_{p}^{(-\mathbb{N})} \times \mathbb{F}_{p}^{\mathbb{N}_{0}}$ and hence is a simple contraction group.

While Theorem A settles the locally compact case, the following problem remains unsolved:

Problem 6.6 Is it true that all Lie contraction groups over a non-locally compact, complete ultrametric field $(\mathbb{K},||$.$) of positive characteristic are$ torsion groups?

It would be enough to prove this for all simple Lie contraction groups over $\mathbb{K}$.

Let us close this section with material concerning Section 5. The following example shows that, in the case of positive characteristic, analytic contraction groups need not be determined by the Lie algebra and its automorphism. Not even the local structure of the group is determined.

Example 6.7 Let $\mathbb{F}$ be a finite field and $\mathbb{K}:=\mathbb{F}((X))$. We set $p:=\operatorname{char}(\mathbb{F})$ and consider the 3 -dimensional $\mathbb{K}$-analytic Lie group $G:=\mathbb{K}^{2} \rtimes_{\beta} \mathbb{K}$, where $\beta: \mathbb{K} \rightarrow \operatorname{Aut}\left(\mathbb{K}^{2}\right), z \mapsto \beta_{z}$ is the homomorphism given by

$$
\beta_{z}(x, y):=\left(x+z^{p} y, y\right) \text { for } x, y, z \in \mathbb{K} .
$$

Then the map

$$
\alpha: G \rightarrow G, \quad(x, y, z) \mapsto\left(X^{p+1} x, X y, X z\right) \quad \text { for } x, y, z \in \mathbb{K}
$$

is a contractive automorphism of $G$. Given $g=(x, y, z)$ and $h=(a, b, c)$ in $G$, we have $f(g, h):=g h g^{-1} h^{-1}=\left(z^{p} b-c^{p} y, 0,0\right)$. Since $\left|z^{p} b\right|=O\left(\|g\|^{2}\right) O(\|h\|)$ as $(g, h) \rightarrow(0,0)$ and $\left|c^{p} y\right|=O\left(\|h\|^{2}\right) O(\|g\|)$, it follows that

$$
f(g, h)=o\left(\|(g, h)\|^{2}\right)
$$


(using Landau's big $O$ and small $o$-notation). The second order Taylor expansion of the commutator map $f$ of the $C^{\omega}$-Lie group $G$ around $(0,0)$ reads

$$
f(g, h)=[g, h]+o\left(\|(g, h)\|^{2}\right)
$$

(cf. item 5 in [27, Part II, Chapter IV, $\S 7]$ ). Comparing with (13), we deduce that $[g, h]=0$ for all $g, h \in \mathbb{K}^{3}$. Thus $L(G)=\mathbb{K}^{3}$ is an abelian Lie algebra. Also, $L(\alpha)$ is the linear map $\gamma: \mathbb{K}^{3} \rightarrow \mathbb{K}^{3},(x, y, z) \mapsto\left(X^{p+1} x, X y, X z\right)$. Now $\left(\mathbb{K}^{3},+\right)$ is a 3 -dimensional $\mathbb{K}$-analytic Lie group admitting $\gamma$ as a contractive $\mathbb{K}$-analytic automorphism. We have $L\left(\mathbb{K}^{3}\right)=L(G)$ and $L(\gamma)=L(\alpha)$. However, $\left(\mathbb{K}^{3},+\right)$ is abelian while $G$ is not (and in fact $G$ does not even have an abelian open subgroup).

One would not expect a positive solution to the following existence question, but the authors currently do not know counterexamples.

Problem 6.8 If $\mathfrak{g}$ is a Lie algebra over a local (or complete ultrametric) field $\mathbb{K}$ of positive characteristic and $\beta: \mathfrak{g} \rightarrow \mathfrak{g}$ a contractive Lie algebra automorphism, can we always find an analytic (or at least $C^{k}$ ) Lie group $G$ and an analytic (or $C^{k}$ ) contractive automorphism $\alpha: G \rightarrow G$ such that $\mathfrak{g}=L(G)$ and $\beta=L(\alpha)$ ?

\section{References}

[1] Baumgartner, U. and G. A. Willis, Contraction groups and scales of automorphisms of totally disconnected locally compact groups, Israel J. Math. 142 (2004), 221-248.

[2] Bertram, W., H. Glöckner and K.-H. Neeb, Differential calculus over general base fields and rings, Expo. Math. 22 (2004), 213-282.

[3] Bourbaki, N., "Variétés différentielles et analytiques. Fascicule de résultats," Hermann, Paris, 1967.

[4] Bourbaki, N., "Lie Groups and Lie Algebras" (Chapters 1-3), Springer-Verlag, 1989.

[5] Bourbaki, N., "Groupes et Algèbres de Lie" (Chapters 7-8), Diffusion C.C.L.S, Paris, 1975. 
[6] Dani S. G. and R. Shah, Contraction subgroups and semistable measures on p-adic Lie groups, Math. Proc. Camb. Phil. Soc. 110 (1991), 299-306.

[7] Dixon, J.D., M.P.F. du Sautoy, A. Mann and D. Segal, "Analytic pro- $p$ Groups," Cambridge Univ. Press, 1991.

[8] Glöckner, H., Scale functions on p-adic Lie groups, Manuscr. Math. 97 (1998), 205-215.

[9] Glöckner, H., Smooth Lie groups over local fields of positive characteristic need not be analytic, J. Algebra 285 (2005), 356-371.

[10] Glöckner, H., Every smooth p-adic Lie group admits a compatible analytic structure, Forum Math. 18 (2006), 45-84.

[11] Glöckner, H., Implicit functions from topological vector spaces to Banach spaces, Israel J. Math. 155 (2006), 205-252.

[12] Glöckner, H., Aspects of p-adic non-linear functional analysis, pp. 237253 in: A. Yu. Khrennikov, Z. Rakić and I. V. Volovich (eds.), p-Adic Mathematical Physics. 2nd International Conference (Belgrade, 2005), AIP Conference Proceedings 826, Amer. Inst. Physics, New York, 2006 (see also arXiv:math/0602081).

[13] Glöckner, H., Finite order differentiability properties, fixed points and implicit functions over valued fields, preprint, arXiv:math/0511218.

[14] Glöckner, H., Stable manifolds for dynamical systems over ultrametric fields, in preparation.

[15] Glöckner, H., Scale functions on Lie groups over local fields of positive characteristic, in preparation.

[16] Glöckner, H., Lie groups of type $R$ over local fields of positive characteristic, in preparation.

[17] Glöckner, H. and G.A. Willis, Uniscalar p-adic Lie groups, Forum Math. 13 (2001), 413-421.

[18] Glöckner, H. and G. A. Willis, Classification of the simple factors appearing in composition series of totally disconnected contraction groups, preprint, arXiv:math/0604062. 
[19] Hazod, W. and S. Siebert, "Stable Probability Measures on Euclidean Spaces and on Locally Compact Groups," Kluwer, Dordrecht, 2001.

[20] Irwin, M. C., On the stable manifold theorem, Bull. London Math. Soc. 2 (1970), 196-198.

[21] Jacobson, N., "Basic Algebra II," W. H. Freeman and Company, New York, 1989.

[22] Mac Lane, S., "Categories for the Working Mathematician," SpringerVerlag, New York, 1994.

[23] Margulis, G.A., "Discrete Subgroups of Semisimple Lie Groups," Springer-Verlag, 1991.

[24] Raja, C. R.E., On classes of p-adic Lie groups, New York J. Math. 5 (1999), 101-105.

[25] Robinson, D. J. S., "A Course in the Theory of Groups," SpringerVerlag, 1996.

[26] Schikhof, W. H., "Ultrametric Calculus," Cambridge University Press, 1984.

[27] Serre, J.-P., "Lie Algebras and Lie Groups," Springer-Verlag, 1992.

[28] Siebert, E., Contractive automorphisms on locally compact groups, Math. Z. 191 (1986), 73-90.

[29] Siebert, E., Semistable convolution semigroups and the topology of contraction groups, pp. 325-343 in: H. Heyer (Ed.), "Probability Measures on Groups IX" (Oberwolfach 1988), 1989.

[30] Wang, J.S.P., The Mautner phenomenon for p-adic Lie groups, Math. Z. 185 (1984), 403-412.

[31] Weil, A., "Basic Number Theory," Springer-Verlag, 1967.

[32] Wells, J. C., Invariant manifolds of non-linear operators, Pacific J. Math. 62 (1976), 285-293.

[33] Willis, G.A., The structure of totally disconnected, locally compact groups, Math. Ann. 300 (1994), 341-363.

Helge Glöckner, TU Darmstadt, Fachbereich Mathematik AG 5, Schlossgartenstr.7, 64289 Darmstadt, Germany. E-Mail: gloeckner@mathematik.tu-darmstadt.de 